\title{
Elsewhere in Romance: Evidence from Clitic Clusters
}

\author{
Diego Pescarini
}

This article focuses on sequences of Romance clitics wherein a pronominal form is replaced by another clitic exponent, which is prima facie morphologically unmotivated. Bonet (1991) and Harris (1994) among others have argued that these synthetic clusters can be due to the insertion of an elsewhere clitic: a default, nonspecified item that is inserted as a last resort whenever the insertion of other clitics is ruled out. In this article, independent pieces of evidence gathered from Italian and Italian dialects are shown to support this hypothesis.

Keywords: Distributed Morphology, clitic clusters, syncretism, elsewhere, Romance languages

\section{Introduction}

Synthetic clusters are clitic sequences wherein a syntactically and semantically opaque pronoun replaces the expected, transparent morphological exponent. For instance, in Italian, when the reflexive clitic si (1a) precedes the homophonous impersonal clitic (1b), it is replaced by $c i$, as shown in $(1 d)$.

$$
\begin{aligned}
& \text { (1) a. Carlo si lava ogni giorno. } \\
& \text { Carlo himself.cL wash.3sg every day } \\
& \text { 'Carlo washes every day.' } \\
& \text { b. La macchina si lava ogni giorno. } \\
& \text { the car.oBJ one.CL wash.3sg every day } \\
& \text { 'The car is washed every day.' } \\
& \text { c. *Si si lava ogni giorno. } \\
& \text { himself/herself.cL one.CL wash.3sG every day } \\
& \text { 'You(imp.) wash every day.' } \\
& \text { d. Ci si lava ogni giorno. } \\
& \text { himself/herself.CL one.CL wash.3sG every day } \\
& \text { 'You(imp.) wash every day.' }
\end{aligned}
$$

Since Bonet's (1991) seminal dissertation, synthetic clusters have normally been accounted for by postsyntactic operations whereby a pronominal form (the target $\mathrm{T}$ ) is replaced by a less marked item (the substitute $\mathrm{S}$ ), leaving the interpretation of the pronoun unchanged.

$$
\text { (2) } \mathrm{T} \rightarrow \mathrm{S}
$$

For comments on previous drafts of this article, I am very grateful to Andrea Calabrese, Jonathan Bobaljik, Andrew Nevins, Paola Benincà, Nicoletta Penello, Anna Cardinaletti, Mair Parry, Andrea Cattaneo, Laura Vanelli, and Cecilia Poletto. 
Sometimes this can be accounted for either by deleting a privative feature (Halle and Marantz's (1993) impoverishment; e.g., $[\mathrm{F}] \rightarrow \emptyset$ ) or by turning a binary feature to the unmarked value (e.g., $[+\mathrm{F}] \rightarrow[-\mathrm{F}]$; see Noyer 1992 , Calabrese 1995). ${ }^{1}$ Both these solutions account for welldocumented cases where, for instance, plural Ts are replaced by singular Ss, feminine by masculine, dual by plural, and so on. However, when a syntactic or semantic relation between $\mathrm{T}$ and $\mathrm{S}$ cannot be proved, impoverishment and feature change cannot capture the substitution properly and, in these cases, $\mathrm{S}$ is normally assumed to be an elsewhere item - that is, a featureless exponent that is inserted by default when a (more) specified Vocabulary item cannot be picked out by manipulating some features via impoverishment or feature change. This proposal is promising but circular, since the elsewhere status of $\mathrm{S}$ does not normally follow from independent evidence. In this article, I will seek to break this circularity and carry out a principled analysis of elsewhere items by discussing some phenomena that allow us to attribute elsewhere status to a particular item.

After briefly describing the Italian clitic system in section 2, I will compare Italian synthetic clusters in section 3 in order to show that, within the same language, different targets are replaced by the same substitute.

(3) $\mathrm{T}_{1}, \mathrm{~T}_{2}, \mathrm{~T}_{3} \rightarrow \mathrm{S}$

I will argue that this pattern is consistent with the elsewhere hypothesis, while other accounts have difficulty in explaining why different environments converge toward insertion of the same S. In section 4 , I will focus on a case where substitution cannot take place and a clitic is obliterated-that is, its syntactic node is deleted (Arregi and Nevins 2006, Calabrese 2008b). Crucially, we will see that the obliterated clitic corresponds to the elsewhere item that, being the last resort, is correctly predicted not to be replaced by any other clitic. In section 5, I will discuss a strong correlation between synthetic clusters and syncretic patterns, showing that the same clitic responsible for synthetic clusters is normally a syncretic element, and I will claim that this correlation directly follows from the elsewhere status of that clitic. Finally, in section 6, I will compare several languages and dialects in order to show that in different languages (L) the same target is replaced by different substitutes, a phenomenon that is difficult to account for within other analyses.

$$
\text { (4) } \begin{aligned}
\mathrm{T} & \rightarrow \mathrm{S}_{1} \text { in } \mathrm{L}_{1} \\
\mathrm{~T} & \rightarrow \mathrm{S}_{2} \text { in } \mathrm{L}_{2} \\
\mathrm{~T} & \rightarrow \mathrm{S}_{3} \text { in } \mathrm{L}_{3}
\end{aligned}
$$

In conclusion, I will show that patterns (2)-(4) are all consistent with the hypothesis that each language selects a specific elsewhere item, whose puzzling distribution I will account for in the following sections.

\footnotetext{
${ }^{1}$ The following data are not conclusive for preferring either of these analyses; for an in-depth comparison of these options, see Calabrese 2008b.
} 


\section{Italian Clitics}

Harris (1991, 1992, 1994) suggests that Romance (pro)nominal elements are formed by a root followed by a thematic vowel marking the nominal class they belong to. According to this analysis, the morphology of Italian clitics (in table 1) is due to the combination of the Vocabulary items in $(5) .^{2}$

(5) a. Roots

$$
\begin{aligned}
& \text { gl- } \leftrightarrow \text { [-participant, - speaker, - plural, -feminine]/[dative] } \\
& \text { l- } \leftrightarrow[\text {-participant, }- \text { speaker }] /[ \pm \text { feminine, } \pm \text { plural }] \\
& \text { m- } \leftrightarrow \text { [+participant, +speaker, - plural] } \\
& \text { t- } \leftrightarrow \text { [+ participant, }- \text { speaker, - plural] } \\
& \text { c- } \leftrightarrow \text { [+participant, +speaker, +plural] } \\
& \text { V- } \leftrightarrow \text { [+participant, - speaker, +plural] } \\
& \text { s- } \leftrightarrow \text { [-participant, - speaker] } \\
& \text { n- } \leftrightarrow \text { [ablative/partitive] } \\
& \mathrm{v}-\leftrightarrow \text { [distal locative] } \\
& \text { c- } \leftrightarrow \text { [locative] }
\end{aligned}
$$

\begin{tabular}{|c|c|c|c|c|c|c|}
\hline & \multicolumn{2}{|c|}{1} & \multicolumn{2}{|c|}{2} & \multicolumn{2}{|c|}{3} \\
\hline & $\mathrm{Sg}$. & Pl. & Sg. & Pl. & $\mathrm{Sg}$. & Pl. \\
\hline Dir. obj. & & & & & lo/la & li/le \\
\hline Indir. obj. & $\mathrm{mi}$ & ci & $\mathrm{ti}$ & vi & gli/le & - \\
\hline Reflexive & & & & & & \\
\hline Partitive & & & & & & \\
\hline Locative & & & & & & \\
\hline
\end{tabular}

b. Thematic vowels

$$
\begin{aligned}
-\mathrm{o} & \leftrightarrow \text { I } \\
-\mathrm{a} & \leftrightarrow \text { II } \\
-\mathrm{e} & \leftrightarrow \text { III } \\
-\mathrm{i} & \leftrightarrow \text { elsewhere }
\end{aligned}
$$

It is worth noting that the inventory in table 1 displays some identical forms: in particular, the locative and the 1 st person plural are both expressed by $c i$; the distal (i.e., distant from speaker) locative $v i$ is identical to the 2 nd person plural; and the feminine $l e$ is the exponent of both the

Table 1

Italian: Paradigm of clitic pronouns

\footnotetext{
${ }^{2}$ The features I will adopt are [ \pm participant] (the reference set contains the speaker and/or the hearer); $[ \pm$ speaker $]$ (the reference set contains the speaker); [ \pm plural] (the reference set contains more than one individual); and case features like [dative], [ablative/partitive], [distal locative], and [locative]. The last four are conventional labels standing for matrices of case features I will not try to analyze here. For a proposal, see Calabrese 1995, 1998, 2008a.
} 
dative singular and the accusative plural clitic. These cases of identity can be interpreted in two ways:

- homophony: two Vocabulary items having identical phonological exponents; or

- syncretism: the same item being inserted in different environments.

Homophony is the zero hypothesis that is assumed in (5), while the possibility of syncretism needs further investigations that will be carried out in sections 5 and 6 .

\section{Italian Synthetic Clusters}

In Italian, several sequences of clitics include a synthetic exponent that displays a mismatch between its morphological form and its syntactic function. Here are the relevant cases:

I. When the feminine dative le precedes a 3rd person clitic, ${ }^{3}$ it must be replaced by the masculine gli $(\rightarrow$ glie / _ l- $)$.

(6) Glie/*Le lo presto.

to.her.CL it.CL lend.1sG

'I lend it to her.'

II. The 3rd person dative ( gli/le) can be replaced by the clitic $c i$ to avoid a violation of the Person-Case Constraint (PCC) (as in Barceloní Catalan; see Bonet 2008 and section 6) — that is, the principle ruling out clitic clusters when the indirect object is 3rd person and the direct object is 1st/2nd person (Bonet 1991).

(7) $\mathrm{Ti}$ ci/*gli presento io, al direttore.

you.CL to.him.CL introduce.1sG I to.the director

'I will introduce you to him (the director).'

It is worth noting that the interpretability of the cluster formed in (7) shows that the corresponding syntactic configuration is licit (namely, interpretable), pace Anagnostopoulou's (2005) and Nevins's (2007) accounts of the PCC.

III. A reflexive clitic preceding an impersonal one is replaced by the clitic $c i$, as pointed out by Cinque (1988), among others. ${ }^{4}$

(8) $\mathrm{Ci} / * \mathrm{Si}$ si lava ogni giorno.

himself/herself.CL one.CL wash.3sG every day

'You(imp.) wash every day.'

${ }^{3}$ The same substitution takes place when le precedes the partitive clitic ne.

(i) Glie/*Le ne presto tre.

to.her.cL of.them.CL lend.1sG three

'I lend her three of them.'

${ }^{4}$ Even though Bonet (1991) assumes that the target is the impersonal, the template in (i) describing the order of Italian clitics suggests that the target should be the reflexive clitic, which precedes the impersonal.

(i) gli $/$ le $>\mathrm{mi}>$ ti $/ \mathrm{vi}>$ ci $>$ si $($ refl. $)>$ lo/la/li/le $>$ si (imp. $)>$ ne 
IV. In clusters formed by an ablative and an identical partitive clitic, the leftmost clitic can be replaced by the clitic $c i$ (in the following glosses, I will assume that the target is the ablative, even if the real order cannot be detected).

(9) $\mathrm{Ce} / * \mathrm{Ne}$ ne escono molti.

from.there.CL of.them.CL come.out.3PL many

'Many of them come out from there.'

To recapitulate, then, the substitutions displayed by Italian are those shown in table 2. Given this scenario, I claim that no single principle or constraint (see proposals by Grimshaw (1997, 2001), Nevins (2007), and Cardinaletti (2008), among others) can trigger all these substitutions; rather, they are due to a constellation of different constraints. Descriptively, I will capture these restrictions with three principles. Restriction I is due to a filter preventing the insertion of items bearing marked gender features.

(10) $*$ Clitic $_{\alpha}+$ clitic $_{\beta}$ if $\alpha$ is $[+$ feminine $]$.

Restriction II is due to the PCC; I will capture this situation with the filter in (11).

(11) $*$ Clitic $_{\alpha}+$ clitic $_{\beta}$ if $\alpha$ is [-participant $]$ and $\beta$ is [+participant $]$.

Restrictions III and IV are triggered by an Obligatory Contour Principle-like constraint preventing the cooccurrence of identical exponents (Grimshaw 1997, 2001).

(12) $*$ Clitic $_{\alpha}+\operatorname{clitic}_{\beta}$ if $\alpha=\beta$.

These filters are simple stipulations that sum up a system of restrictions that I will not address here. Rather, in what follows I will mainly focus on the complementary issue - the morphology of the replacing item - within the framework sketched in section 1. Following Calabrese (2008b), pattern $\mathrm{I}$ is a case of feature change whereby the gender feature turns to its unmarked value, allowing insertion of the masculine item gli instead of le. On the other hand, patterns II-IV cannot be accounted for by a similar feature manipulation because they do not depend on a clear pivotal

Table 2

Italian: List of synthetic clusters

\begin{tabular}{|c|c|c|c|}
\hline & Rule & Description & Ex. \\
\hline I & le $\rightarrow$ gli $/$ _ lo, la, li, le, ne & $\begin{array}{l}\text { The feminine dative le is replaced by the } \\
\text { masculine gli before } l o, l a, l i, l e, n e \text {. }\end{array}$ & (6) \\
\hline II & $\mathrm{gli} / \mathrm{le} \rightarrow \mathrm{ci} /$ mi, ti, vi & $\begin{array}{l}\text { The } 3 \text { rd person dative (gli/le) is replaced } \\
\text { by } c i \text { before a } 1 \text { st } / 2 \text { nd person clitic. }\end{array}$ & (7) \\
\hline III & $\mathrm{si} \rightarrow \mathrm{ci} / \ldots \mathrm{si}$ & $\begin{array}{l}\text { The reflexive clitic } s i \text { is replaced by } c i \\
\text { before the impersonal } s i \text {. }\end{array}$ & (8) \\
\hline IV & ne $\rightarrow$ ci / — ne & $\begin{array}{l}\text { The ablative } n e \text { is replaced by } c i \text { before } \\
\text { the partitive } n e \text {. }\end{array}$ & (9) \\
\hline
\end{tabular}


feature and, crucially, in those cases three different clitics ( $g l i, n e, s i)$ are replaced by the same item $(c i)$.

(13) $\mathrm{T}_{1}, \mathrm{~T}_{2}, \mathrm{~T}_{3} \rightarrow \mathrm{S}$

Given the pattern in (13), the stipulation of (at least) three language-specific operations that change the features of three targets and finally select the same substitute is an ad hoc solution because, assuming any inventory of features and a list of (recursive) feature change operations, we will overgenerate a large number of possible repairs for each target. As a consequence, it is unlikely that three forms in the same language are replaced by the same substitute via feature change or impoverishment.

An alternative is to follow Harris $(1994,1997)$ and (in part) Bonet $(1991,1995)$ in claiming that patterns II-IV are due to insertion of the elsewhere item $c i$ as a last resort. At present, this hypothesis also does not reach explanatory adequacy, but-unlike the previous one-it allows us to formulate and verify a series of empirical predictions, which I will address in the following sections.

Finally, if this hypothesis is confirmed, the analysis of Italian clusters will show that morphological substitutions are due both to feature change operations (I) and to elsewhere insertion (II-IV). Moreover, since le is replaced by gli (I), which in turn is replaced by $c i$ (II), these repairs must be ranked: elsewhere insertion takes place after the morphological component fails to pick out a proper item via feature change. I will return to this point at the end of the next section.

\section{Obliteration}

Supposing that each morphological inventory includes an elsewhere item, what happens when this item is itself targeted by a constraint blocking its insertion? The elsewhere item cannot be replaced via feature change because it is featureless; therefore, Vocabulary Insertion does not take place at all because the morphological component is not able to select a candidate; as a result, a position in the cluster is expected to be obliterated (Arregi and Nevins 2006) and left void.

(14) $\mathrm{T} \rightarrow \emptyset$ if $\mathrm{T}$ is the elsewhere item

Italian is consistent with this prediction. For instance, we have seen that the double occurrence of the same clitic is ruled out, probably by an OCP-like constraint. Crucially, if the clitic violating the OCP is the elsewhere item $c i$, the sentence is grammatical only if a clitic is dropped.

(15) $(* \mathrm{Ci})$ ci porta Micol. there.CL us.CL bring.3sG Micol

'Micol brings us there.'

This pattern provides relevant evidence for the proposal that $c i$ is the Italian elsewhere item.

It is worth noting that obliteration is allowed when the locative clitic is optional, as in (15)_namely, when it resumes an adjunct PP. By contrast, obliteration fails when the clitic 
resumes an argument. We have already seen that the elsewhere item $c i$ can replace the dative clitic (both gli and le) to avoid violating the PCC. But if the direct object is 1st person plural, the sentence will then have a cluster formed by two identical clitics, a configuration that violates the OCP.
*Micol ci
ci ha presentato.
Micol to.him/her.CL us.CL has introduced
'Micol has introduced us to him/her.'

One clitic is therefore expected to be dropped (obliterated), but here-unlike in (15) — obliteration does not make the sentence grammatical.
(17) *Micol (ci)
ci ha presentato.
Micol to.him/her.cL us.CL has introduced
'Micol has introduced us to him/her.'

The configuration in (16) is irreparable, because obliteration cannot target arguments like the indirect object of (17). On the other hand, the configuration in (15) can be repaired because obliteration can drop locative adjuncts.

This discussion sheds light on the behavior of Spanish, where, according to Harris (1994), the reflexive/impersonal clitic se is the elsewhere clitic. In fact, it replaces the dative le (pl. les), giving rise to the well-known "spurious $s e$ " pattern.
(18) Juan se/*le
lo compró.
Juan to.him/her.CL/to.them.CL it.CL bought
'Juan bought it for him/her/them.'

Moreover, as with Italian *si si, a cluster formed by two instances of se, reflexive and impersonal, is ungrammatical.

(19) *Cuando se come, se se lava las manos antes. when one.CL eat.3sG himself/herself.CL one.CL wash.3sG the hands before 'You(imp.) wash your hands before eating.'

The ungrammaticality of this sentence is consistent with both Harris's analysis and my proposal: the cluster is irreparable because, se being the elsewhere item, the morphological component cannot select any less-specified element in order to avoid violating the OCP. On the other hand, the clitic position cannot be obliterated because both clitics refer to an argument.

In conclusion, the data I have discussed so far are consistent with a model of Vocabulary Insertion involving three ranked repairs: feature change, elsewhere insertion, and obliteration. As shown schematically in figure 1, when a clitic is targeted by a constraint, the morphological component first tries to identify an alternative candidate via feature manipulation. If no such candidate is available, it inserts the elsewhere item. Finally, if the elsewhere item is also ruled out, the clitic position is obliterated, when possible. 
Vocabulary Insertion:

Select the best Vocabulary item

(Halle and Marantz 1993)

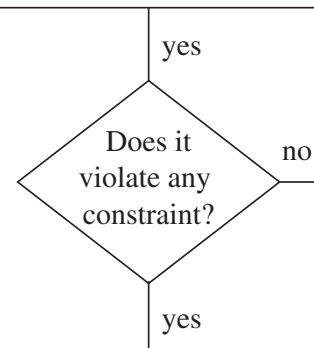

Manipulate its feature in order to select another candidate

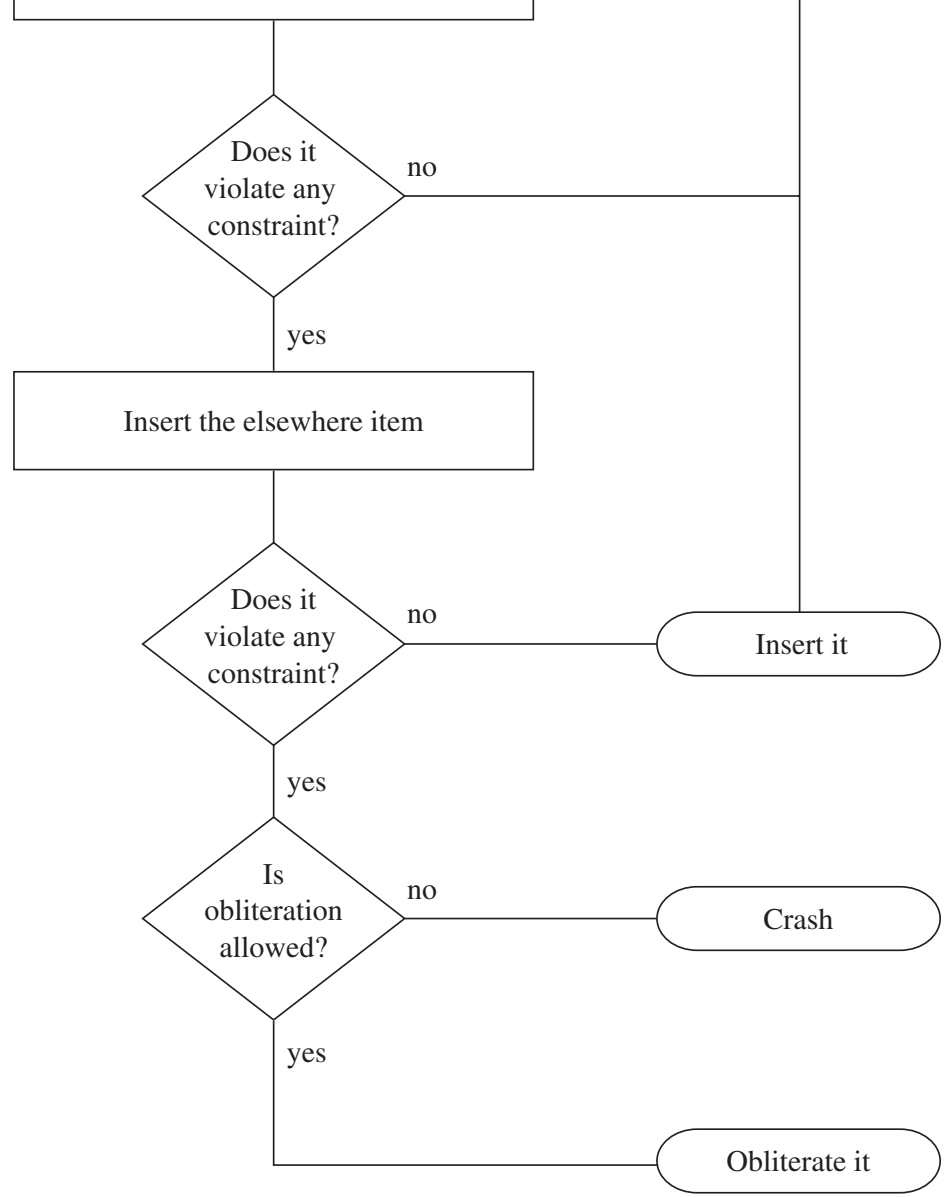

\section{Figure 1}

Model of Vocabulary Insertion 


\section{An Aside on Syncretism}

A syncretic clitic is a morphological exponent that, in a given language, shows up in several syntactic contexts, where other languages insert different items. For instance, in Italian the same exponent $c i$ is a 1 st person plural pronoun (20a) and a locative form (20b), whereas, in the corresponding sentences, the dialect spoken in Verona displays two different exponents: $n e$ for the 1 st person $((21 \mathrm{a})=(20 \mathrm{a}))$ and $g h e / g e /$ for the locative $((21 \mathrm{~b})=(20 \mathrm{~b}))$.

(20) a. Ci porta Micol.

(Italian)

us.CL bring.3sG Micol

'Micol brings us.'

b. $\mathrm{Ci}$ vado in macchina.

there.CL go.1sG by car

'I go there by car.'

(21) a. La ne porta la Micol

(Veronese)

she.CL us.CL bring.3sg the Micol

'Micol brings us.'

b. Ghe vao in machina.

there.CL go.1sG by car

'I go there by car.'

While synthetic clusters are due to contextual substitution, syncretism is the outcome of a diachronic process that makes the etymological target fall out of use. In Italian, for instance, the etymological form of the 1 st person plural (no, ne $<$ Lat. NOS), which is rarely attested, appearing in just a few thirteenth-century documents (Rohlfs 1969, Cardinaletti and Egerland, to appear), has been replaced by the syncretic item $c i$.

Crucially, this syncretic item $(\mathrm{ci})$ corresponds to the elsewhere clitic displayed by synthetic clusters; see section 3. This correspondence directly follows from the hypothesis adopted here: the elsewhere item being an unspecified form, it will replace other items both synchronically and diachronically. ${ }^{5}$ Given this tight and principled correlation between these phenomena, syncretism provides a further diagnostic for detecting elsewhere items: an elsewhere clitic is therefore expected to be a syncretic exponent (if a language displays any pattern of syncretism).

This is why, before carrying out a crosslinguistic analysis of both syncretism and synthetic clusters in the next section, I will briefly sketch the main syncretic patterns found in (Italo-) Romance. According to data provided by Rohlfs (1969) and Manzini and Savoia (2005), the whole picture can be captured by assuming three types of substitutes (locative, partitive, and 3rd

\footnotetext{
${ }^{5}$ The data do not show any clear causal connection between these phenomena. Therefore, I cannot reject either of these alternative explanations:

1. Syncretism is due to the elsewhere item. Syncretism has been caused by the same process of substitution responsible for synthetic clusters, namely, the retreat to the elsewhere exponent. According to this analysis, in Old Italian the etymological 1st person plural pronoun was replaced by the elsewhere item $c i$, giving rise to the syncretism in (20). This is the standard Distributed Morphology account of syncretism discussed by, among others, Bobaljik (2002).

2. The elsewhere item is due to syncretism. Historical processes have neutralized several grammatical distinctions, giving rise to a default case that, in Italian, is the locative. An analysis of case syncretism consistent with this hypothesis has been discussed by, among others, Calabrese (2008a).
} 


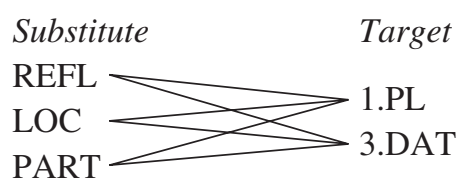

Figure 2

Patterns of substitution in (Italo-)Romance

person reflexive), which replace two preferred targets (1st person plural and 3rd person dative clitic), as shown schematically in figure 2. For instance, the locative has replaced the 3rd person dative clitic in several dialects of southern Italy (Puglia, Calabria, Campania, Sicily) and the 1st person plural pronoun in many central dialects (e.g., Florentine). The reflexive marker has replaced the 3rd person dative clitic in several dialects of Calabria (Rohlfs 1969:156n1, Pescarini 2009) and the reflexive form of the 1st person plural pronoun in many dialects of central and northern Italy (Roma, Northern Toscana, Emilia-Romagna, Veneto, Lombardia; see Benincà and Poletto 2004). The partitive has replaced the 3rd person dative clitic and/or the 1 st person plural pronoun in other southern dialects (Calabrese 1995, Loporcaro 1995, 2002). According to the hypotheses above, these patterns entail that the reflexive, the locative, and the partitive are the elsewhere items of these Italian dialects; indeed, in the next section I will show that these substitutes normally correspond to the elsewhere clitic inserted in synthetic clusters.

\section{Crosslinguistic Evidence}

As the purpose of this section is largely comparative, I will not provide an analysis as fine as the one I gave earlier for Italian. Rather, I will mainly highlight some crosslinguistic similarities, leaving an in-depth analysis of each dialect for further research. In particular, the data will confirm the following predictions about the behavior of the elsewhere item:

1. The elsewhere item replaces different targets (see section 3).

2. The elsewhere item cannot be replaced; it can only be obliterated (see section 4).

3. The elsewhere item corresponds to a syncretic element, if present (see section 5).

First, I will discuss a spurious se pattern found in the Sardinian dialect spoken in Sarroch (province of Cagliari), where the dative clitic $d i$ is replaced by si when clustered.

(22) a. di

pottu unu libru.

to.him/her.CL bring.1sG a book

'I bring him/her a book.'

b. si/*di du pottu.

to.him/her.CL it.CL bring.1sG

'I bring it to him/her.'

Crucially, the Sarroch clitic inventory, unlike the Spanish one, shows a straightforward case of syncretism that confirms Prediction 3: as shown in table 3, the clitic si is indeed used as the 3rd 
Table 3

Sarroch dialect: Paradigm of clitic pronouns

\begin{tabular}{|c|c|c|c|c|c|c|}
\hline & \multicolumn{2}{|c|}{1} & \multicolumn{2}{|c|}{2} & \multicolumn{2}{|c|}{3} \\
\hline & $\mathrm{Sg}$. & Pl. & Sg. & Pl. & Sg. & Pl. \\
\hline Dir. obj. & & & & & $\mathrm{du} / \mathrm{da}$ & dus/das \\
\hline Indir. obj. & $\mathrm{mi}$ & si & ti & si & & \\
\hline Reflexive & & & & & & \\
\hline Partitive & & & & $\mathrm{di}$ & & \\
\hline Locative & & & & & & \\
\hline
\end{tabular}

person reflexive and the 1 st and 2 nd person plural object. This distribution can easily be derived from the inventory in (23), where si is the elsewhere element (class markers are not taken into consideration for reasons of space).

(23) d- $\leftrightarrow[-$ participant, - speaker $] /[ \pm$ feminine, \pm plural $]$

m- $\leftrightarrow[+$ participant, +speaker, +singular $]$

t- $\leftrightarrow[+$ participant, - speaker, + singular]

nd- $\leftrightarrow$ [partitive]

c- $\leftrightarrow$ [locative]

s- $\leftrightarrow[$ ]

Finally, the cooccurrence of two si markers is ruled out, as in Spanish, in accordance with Prediction 2. In conclusion, a series of consistent pieces of evidence shows that the clitic si is the elsewhere item of the Sardinian dialect spoken in Sarroch and, mutatis mutandis, this result also supports Harris's (1994) analysis of Spanish discussed in section 4.

Second, I will discuss a series of dialects that-like Italian-display an elsewhere clitic corresponding to the locative item. For instance, the Vailate dialect (spoken in the province of Cremona, northern Italy) shows a clear case of syncretism since the clitic ga is used as locative, 1st person plural, and 3rd person dative (see table 4). In this dialect, a cluster formed by the reflexive and the impersonal clitic is expressed by the sequence ga sa (see (24a)), instead of the transparent sequence $s a s a$ : the reflexive clitic is therefore expressed by an opaque exponent corresponding to the syncretic clitic ga (see Prediction 3). Moreover-as in Italian-sequences formed by two instances of $g a$ are not grammatical and are repaired via obliteration (see (24b)), as forecast by Prediction 2 .

(24) a. sa + sa $\rightarrow$ ga sa (*sa sa)

himself/ one.CL

herself.CL

b. ga + ga $\rightarrow$ ga $\left({ }^{*}\right.$ ga ga)

there.CL Us.CL 
Table 4

Vailate dialect: Paradigm of clitic pronouns

\begin{tabular}{|c|c|c|c|c|c|c|}
\hline & \multicolumn{2}{|c|}{1} & \multicolumn{2}{|c|}{2} & \multicolumn{2}{|c|}{3} \\
\hline & Sg. & Pl. & Sg. & Pl. & $\mathrm{Sg}$. & Pl. \\
\hline Dir. obj. & \multirow{2}{*}{$\mathrm{ma}$} & \multirow{2}{*}{ ga } & \multirow{3}{*}{ ta } & \multirow{3}{*}{ va } & $\mathrm{al} / \mathrm{la}$ & $\mathrm{i} / \mathrm{le}$ \\
\hline Indir. obj. & & & & & & \\
\hline Refl./Imp. & & sa & & & & \\
\hline Partitive & \multicolumn{6}{|c|}{ na } \\
\hline Locative & \multicolumn{6}{|c|}{ ga } \\
\hline
\end{tabular}

The same pattern can be found in some dialects of northern Puglia and Molise (especially in the province of Campobasso; see Rohlfs 1969:185-186), such as Poggio Imperiale, S. Paolo di Civitate, Pàstena, and Monteroduni (data from Manzini and Savoia 2005:135-138), where the elsewhere clitic $c e$ is even more pervasive than in Vailate since it is used as impersonal, locative, and 1st person plural, and as the reflexive form for 3rd and 2nd person plural (see table 5). ${ }^{6}$ According to Prediction 2, it is not surprising that when the 3rd person dative $i$ cooccurs with the 3 rd person accusative clitic, it is replaced by the syncretic clitic $c ə$.

(25) $\mathrm{C} / * \mathrm{I} \quad \mathrm{u}$ da.

to.him/her.CL it.CL give.3sG

'He/She gives it to him/her.'

Manzini and Savoia (2005:141) notice a slightly different pattern of syncretism in some dialects of Abruzzi and Molise (e.g., in Vastogirardi and Roccasicura), which display two syncretic exponents: $c ə$ ( $<$ Lat. INCE) and zə (< Lat. SE) (see table 6). In these dialects, the clitic $c ə$ - which is a syncretic exponent (see Prediction 3) — replaces the 3rd person dative form ( $r e$ ) when clustered with an identical accusative clitic.

Table 5

Poggio Imperiale: Paradigm of clitic pronouns (from Manzini and Savoia 2005)

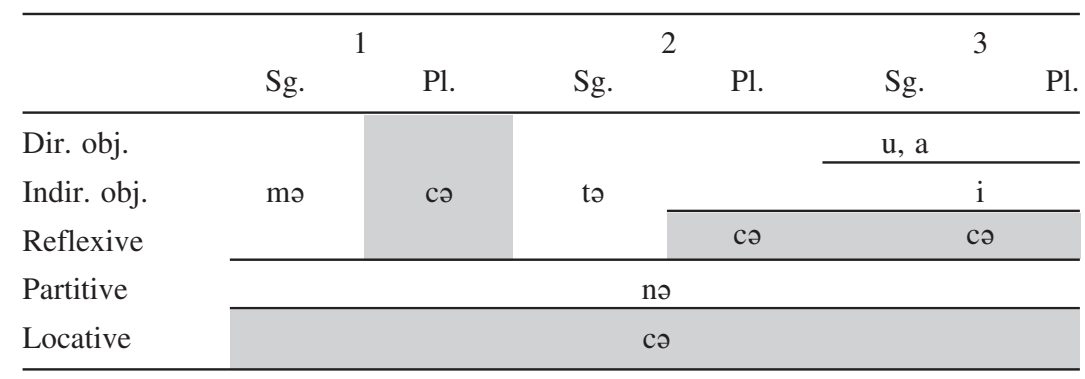

${ }^{6}$ The paradigm is not complete because it is based on sets of examples from Manzini and Savoia 2005:135. 
Table 6

Roccasicura dialect: Paradigm of clitic pronouns

\begin{tabular}{|c|c|c|c|c|c|c|}
\hline & \multicolumn{2}{|c|}{1} & \multicolumn{2}{|c|}{2} & \multicolumn{2}{|c|}{3} \\
\hline & $\mathrm{Sg}$. & Pl. & $\mathrm{Sg}$. & Pl. & Sg. & Pl. \\
\hline Dir. obj. & & CO & & & rə & \\
\hline Indir. obj. & mə & & to & və & & \\
\hline Reflexive & & zo & & & & \\
\hline Partitive & & & & & & \\
\hline Locative & & & & & & \\
\hline
\end{tabular}

(26) rə $\quad+$ rə $\rightarrow$ cə rə (*re re)

to.him/her.CL him/her.CL

Finally, many clusters in Barceloní-the Catalan dialect spoken in Barcelona and analyzed in depth by Bonet $(1991,1995,2008)$ — can be accounted for by suggesting that the locative /i/ - written $h i$-is the default exponent. It replaces en (partitive and/or ablative) when it cooccurs with an identical en exponent or when it is clustered with a 3rd person clitic. (It is worth noting that these are the same contexts triggering the substitutions of Italian-but, whereas the target clitic is on the left of the cluster in Italian, in Barceloní it is on the right.)

(27) a. El jersei, el trauré de l'armari després. the sweater it.CL take.1sG.FuT from the closet later
b. De l'armari, en trauré
el jersei després. from the closet from.there.cL take.1sG.FuT the sweater later

c. El jersei, de l'armari 1 i/*en trauré després. the sweater from the closet it.CL from.there.CL take.1sG.FUT later 'I will take the sweater from the closet later.'

(28) a. Del cine, en van sortir tres nens. from.the cinema from.there.CL came.out.3PL three children

b. De nens, en van sortir tres del cine. of children of.them.cL came.out.3PL three from.the cinema

c. Del cine, de nens $n$ i/*en van sortir tres. from.the cinema of children of.them.CL from.there.CL came.out.3PL three 'Three children came out from the cinema.'

Moreover, the elsewhere $h i$ can replace the dative $l i$ when it violates the PCC (Bonet 1991:209, 2008); this is similar to the Italian case discussed in section 3.

(29) A en Pere m' hi/*li va recomanar en Josep. to the Pere me.CL to.him.CL recommended.3sG the Josep 'Josep recommended me to him (Pere).' 
Finally, hi replaces the 3rd person clitic ho /u/ when it is clustered with the partitive pronoun (Harris 1997). These data show that $h i$ can replace different clitic forms (li, en, and ho), in accordance with Prediction 1. At the same time, the locative $h i$ is always deleted when clustered with a dative $l i$. This fact can be interpreted as a case of obliteration, consistent with Prediction 2 , on the basis of Bonet's analysis suggesting that the clitic $l i$ is formed by a 3rd person exponent $l$ - followed by the oblique marker $-i$, which is syncretic with the locative $h i$ /i/. Therefore, a sequence formed by a dative $/ 1 /+/ \mathrm{i} /$ and a locative /i/ is targeted by the OCP-like constraint causing obliteration of the elsewhere exponent.

(30) a. A Montserrat, hi faré un regal a la Gemma. in Montserrat there.CL give.1sG.FuT a present to the Gemma

b. A la Gemma, li faré un regal a Montserrat. to the Gemma to.her.CL give.1sG.FUT a present in Montserrat

c. A la Gemma, a Montserrat, li (*hi) faré un regal. to the Gemma in Montserrat to.her.CL there.CL give.1sG.FuT a present 'In Montserrat, I will give a present to Gemma.'

To recap, then, even if Barceloní does not display a clear case of syncretism allowing the verification of Prediction 3, its clusters support both Predictions 1 and 2 because the elsewhere $h i$ replaces different items (as shown in (26)-(29)) and is obliterated when targeted by the OCP (as shown in (30)).

So far, I have described dialects displaying an elsewhere item corresponding to either the reflexive or the locative clitic. According to the schema of syncretism in figure 2, I should now focus on the third option: dialects displaying a partitive elsewhere item. However, for both historical and structural reasons this pattern is quite uncommon. First of all, it is worth noting that the schema in figure 2 is somewhat misleading because it does not distinguish between true syncretism and a superficial homophony (see section 2). In fact, as pointed out by Loporcaro (1995, 2002), among others, frequently the partitive clitic $(<$ Lat. INDE) is not a true syncretic clitic, but is simply homophonous (phonologically identical) with the 1 st person plural ( $<$ Lat. NOS). According to this historical observation, dialects like the one spoken in Arce (Lazio region; see table 7)

\section{Table 7}

Arce dialect: Paradigm of clitic pronouns

\begin{tabular}{|c|c|c|c|c|c|c|}
\hline & \multicolumn{2}{|c|}{1} & \multicolumn{2}{|c|}{2} & \multicolumn{2}{|c|}{3} \\
\hline & $\mathrm{Sg}$. & Pl. & $\mathrm{Sg}$. & Pl. & $\mathrm{Sg}$. & Pl. \\
\hline Dir. obj. & \multirow{3}{*}{ me } & ne & \multirow{3}{*}{ te } & \multirow{3}{*}{ ve } & glie/la & glie/le \\
\hline Indir. obj. & & \multirow{2}{*}{ ce } & & & \multicolumn{2}{|c|}{ glie } \\
\hline Reflexive & & & & & \multicolumn{2}{|c|}{ se } \\
\hline Partitive & & & & & & \\
\hline Locative & \multicolumn{6}{|c|}{ ce } \\
\hline
\end{tabular}


display only one true syncretic exponent $(\mathrm{ci})$ and two homophonous clitics $n e$, as represented in the inventory fragment in (31).

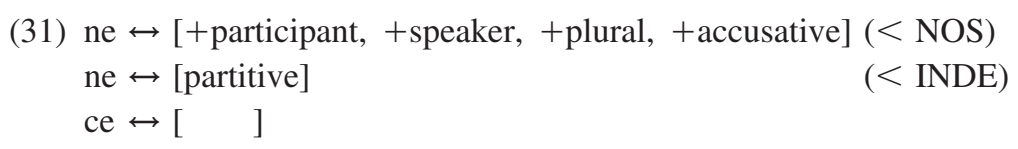

In fact, the synthetic clusters of the Arce dialect confirm that the elsewhere item is $c e$ : it replaces the dative form, according to Prediction 3 (see (32a)), and, according to Prediction 2, two instances of ce cannot cooccur (see (32b)).

(32) a. glie $\quad+$ glie $\rightarrow$ ce glie (*glieglie) to.him/her.CL him/it.CL

b. glie $\quad+$ ne $\rightarrow$ ce ne (*gliene) to.him/her.CL of.them.CL

Traces of an elsewhere item corresponding to the partitive clitic can be found only in some southern dialects (in the regions of Puglia and Calabria), which display the same distribution found in Arce, but with a different morphology of synthetic clusters. The example in (33), for instance, shows that in the dialect of Rocca Imperiale (from Manzini and Savoia 2005:291), the partitive clitic $n$ - replaces the dative clitic (Prediction 3).

(33) $\mathrm{i} \quad+\quad \mathrm{i} \quad \rightarrow$ ni $(* \mathrm{ii})$
to.him/her.CL them.CL

In the same dialect, the clitic no is obliterated when it is followed by an identical partitive clitic, according to Prediction 2.
(34) a. mə nə
me.CL of.them.CL
b. to nə
you.SG.CL of.them.CL
$\begin{array}{ll}\text { c. və } & \text { nə } \\ \text { you.PL.CL } & \text { of.them.CL }\end{array}$
$\begin{array}{cl}\text { d. (*nə) } & \text { nə } \\ \text { us.CL } & \text { of.them.CL }\end{array}$

This pattern shows that, even if uncommon, there are a few cases of an elsewhere clitic of the type ne, which etymologically corresponds to the partitive item. The rarity of this pattern is not due to an accidental gap. Rather, the partitive/ablative item is intrinsically more marked than the locative; that is, it corresponds to a richer feature bundle. This claim is supported by two kinds of evidence. First, there are dialects-like Barceloní (see (28))—where the ablative is replaced by the locative, while the contrary substitution is never attested.

(35) ablative $(* \leftarrow) \rightarrow$ locative 
Second, in many Italian dialects - for instance, in Verona (Veneto region) - the partitive clitic is marked by a compound exponent (ghene) that includes the locative form (ghe). These phenomena suggest that the feature bundle corresponding to the partitive item normally includes the feature configuration that determines the insertion of the locative exponent, which, being less specified, is therefore a better candidate for having elsewhere status.

In conclusion, this sketchy and fragmentary survey of several Romance dialects shows that languages display different elsewhere items that, in the sample dealt with here, correspond to the 3rd person reflexive, the locative, and - rarely - the partitive. These data entail that in different languages the same target is replaced by different substitutes, as summarized in (36).

$$
\text { (36) } \begin{aligned}
\mathrm{T} & \rightarrow \mathrm{S}_{1} \text { in } \mathrm{L}_{1} \\
\mathrm{~T} & \rightarrow \mathrm{S}_{2} \text { in } \mathrm{L}_{2} \\
\mathrm{~T} & \rightarrow \mathrm{S}_{3} \text { in } \mathrm{L}_{3}
\end{aligned}
$$

This pattern cannot depend on any feature-changing strategy, as such a strategy is expected to apply crosslinguistically, giving rise to the same substitution in different languages. Rather, the pattern in (36) is consistent with the presence of a specific elsewhere item in the functional lexicon of each language.

\section{Conclusions}

In this article, I have explored the morphology of synthetic clusters in some Romance dialects. Following Bonet (1991), among others, I have assumed that these phenomena are due to a process of morphological substitution that, in a given language $\mathrm{L}$, replaces a target clitic $\mathrm{T}$ with a substitute $\mathrm{S}$. In particular, I have focused on some cases where $\mathrm{T}$ is supposed to be replaced by a featureless, elsewhere item. I have tested this hypothesis on a wider set of data gathered from Standard Italian, Italian dialects, Spanish, and Barceloní Catalan.

First, I have focused on the synthetic clusters of Italian, showing that in those contexts several clitics are replaced by the same item.

(37) $\mathrm{T}_{1}, \mathrm{~T}_{2}, \mathrm{~T}_{3} \rightarrow \mathrm{S}$

Second, I have discussed a particular case of obliteration (Arregi and Nevins 2006) that, crucially, involves $\mathrm{S}$, which is dropped when targeted by an OCP-like constraint.

(38) $\mathrm{S} \rightarrow \emptyset$

For instance, if the clitic violating the OCP is the Italian elsewhere $c i$, it must be dropped.

(39) $(* \mathrm{Ci})$ ci porta Micol. there.CL us.CL bring.3sG Micol

'Micol brings us there.'

Third, I have observed that $\mathrm{S}$ is normally also a syncretic clitic; and finally, I have shown that $\mathrm{S}$ is subject to crosslinguistic variation: different languages replace the same target with different, language-specific elsewhere items. 


$$
\text { (40) } \begin{aligned}
\mathrm{T} & \rightarrow \mathrm{S}_{1} \text { in } \mathrm{L}_{1} \\
\mathrm{~T} & \rightarrow \mathrm{S}_{2} \text { in } \mathrm{L}_{2} \\
\mathrm{~T} & \rightarrow \mathrm{S}_{3} \text { in } \mathrm{L}_{3}
\end{aligned}
$$

For instance, in Standard Italian, in Barceloní Catalan, and in the dialects of Vailate, Poggio Imperiale, Roccasicura, and Arce, the elsewhere item corresponds to the locative exponent; in Spanish and the Sardinian dialect spoken in Sarroch, the elsewhere item is the reflexive clitic; and in the dialect of Rocca Imperiale, it is the partitive item.

These data support the hypothesis that $\mathrm{S}$ is a language-specific elsewhere clitic that is inserted by default when other items are independently ruled out and feature manipulation is not enough to select a better (namely, more specified) candidate. I have therefore argued for a model of Vocabulary Insertion whereby insertion of the elsewhere clitic follows feature change operations (namely, it takes place when feature change operations are not able to select an alternative candidate) and precedes obliteration (a process that still requires further investigation).

\section{References}

Anagnostopoulou, Elena. 2005. Strong and weak person restrictions: A feature checking analysis. In Clitic and affix combinations, ed. by Lorie Heggie and Fernando Ordóñez, 199-235. Amsterdam: John Benjamins.

Arregi, Karlos, and Andrew Nevins. 2006. Obliteration vs. impoverishment in the Basque g-/z- constraint. In Proceedings of the 30th Annual Penn Linguistics Colloquium, ed. by Tatjana Scheffler, Joshua Tauberer, Aviad Eilam, and Laia Mayol, 1-14. Penn Working Papers in Linguistics 13.1. Philadelphia: University of Pennsylvania, Penn Linguistics Club.

Benincà, Paola, and Cecilia Poletto. 2004. The third dimension of person features. In Syntax and variation, ed. by Leonie Cornips and Karen P. Corrigan, 265-301. Amsterdam: John Benjamins.

Bobaljik, Jonathan David. 2002. Syncretism without paradigms: Remarks on Williams 1981, 1994. Yearbook of Morphology 2001:53-85.

Bonet, Eulàlia. 1991. Morphology after syntax: Pronominal clitics in Romance. Doctoral dissertation, MIT, Cambridge, MA.

Bonet, Eulàlia. 1995. Feature structure of Romance clitics. Natural Language and Linguistic Theory 13: 607-647.

Bonet, Eulàlia. 2008. The Person-Case Constraint and repair strategies. In Agreement restrictions, ed. by Roberta D’Alessandro, Susann Fischer, and Gunnar Hrafn Hrafnbjargarson, 103-128. Berlin: Walter de Gruyter.

Calabrese, Andrea. 1995. Syncretism phenomena in the clitic systems of Italian and Sardinian dialects and the notion of morphological change. In NELS 25: Proceedings of the North East Linguistic Society, ed. by Jill N. Beckman, 151-174. Amherst: University of Massachusetts, Graduate Linguistic Student Association.

Calabrese, Andrea. 1998. Some remarks on the Latin case system and its development in Romance. In Theoretical analyses on Romance languages: Selected papers from the 26th Linguistic Symposium on Romance Languages (LSRL XXVI), Mexico City, 28-30 March, 1996, ed. by José Lema and Esthela Treviño, 71-126. Amsterdam: John Benjamins.

Calabrese, Andrea. 2008a. On absolute and contextual syncretism. In Inflectional identity, ed. by Asaf Bachrach and Andrew Nevins, 156-205. Oxford: Oxford University Press.

Calabrese, Andrea. 2008b. On the shyness of the first person: Investigations on markedness and underspecification in morphology. Paper presented at MUMSA (Markedness and Underspecification in the 
Morphology and Semantics of Agreement), Harvard University, 29 February-2 March. Available at http://homepages.uconn.edu/ anc02008/Papers/On\%20markedness\%20and\%20underspecification\% 20in\%20Morphology-HO.pdf.

Cardinaletti, Anna. 2008. On different types of clitic clusters. In The Bantu-Romance connection, ed. by Cécile De Cat and Katherine Demuth, 41-82. Amsterdam: John Benjamins.

Cardinaletti, Anna, and Verner Egerland. To appear. I pronomi personali e riflessivi. In Grammatica dell'Italiano antico, ed. by Lorenzo Renzi and Giampaolo Salvi. Bologna: Il Mulino.

Cinque, Guglielmo. 1988. On si constructions and the theory of arb. Linguistic Inquiry 19:521-581.

Grimshaw, Jane. 1997. The best clitic: Constraint conflict in morphosyntax. In Elements of grammar, ed. by Liliane Haegeman, 169-196. Dordrecht: Kluwer.

Grimshaw, Jane. 2001. Optimal clitic positions and the lexicon in Romance clitic systems. In Optimalitytheoretic syntax, ed. by Géraldine Legendre, Jane Grimshaw, and Sten Vikner, 205-240. Cambridge, MA: MIT Press.

Halle, Morris, and Alec Marantz. 1993. Distributed Morphology and the pieces of inflection. In The view from Building 20, ed. by Kenneth Hale and Samuel Jay Keyser, 111-176. Cambridge, MA: MIT Press.

Harris, James. 1991. The exponence of gender in Spanish. Linguistic Inquiry 22:27-62.

Harris, James. 1992. The form classes of Spanish substantives. Yearbook of Morphology 1991:65-88.

Harris, James. 1994. The syntax-phonology mapping in Catalan and Spanish clitics. In Papers on phonology and morphology, ed. by Andrew Carnie and Heidi Harley, with Tony Bures, 321-353. MIT Working Papers in Linguistics 21. Cambridge, MA: MIT, MIT Working Papers in Linguistics.

Harris, James. 1997. Why n'ho is pronounced [li] in Barceloní Catalan. In PF: Papers at the interface, ed. by Benjamin Bruening, Yoonjung Kang, and Martha McGinnis, 451-479. MIT Working Papers in Linguistics 30. Cambridge, MA: MIT, MIT Working Papers in Linguistics.

Loporcaro, Michele. 1995. Un capitolo di morfologia storica italo-romanza: It. ant. $n e$ 'ci' e forme meridionali congeneri. L'Italia Dialettale 58:1-48.

Loporcaro, Michele. 2002. External and internal causation in morphological change: Evidence from ItaloRomance dialects. In Morphology 2000, ed. by Sabrina Bendjaballah, Wolfgang U. Dressler, Oskar E. Pfeiffer, and Maria D. Voeikova, 227-240. Amsterdam: John Benjamins.

Manzini, Maria Rita, and Leonardo Savoia. 2005. I dialetti italiani e romanci: Morfosintassi generativa. Alessandria: Edizioni dell'Orso.

Nevins, Andrew. 2007. The representation of third person and its consequences for person-case effects. Natural Language and Linguistic Theory 25:273-113.

Noyer, Rolf. 1992. Features, positions and affixes in autonomous morphological structure. Doctoral dissertation, MIT, Cambridge, MA.

Pescarini, Diego. 2009. I pronomi clitici. In Studi sui dialetti della Calabria, ed. by Diego Pescarini, 63-76. Padua: Unipress.

Rohlfs, Gerhard. 1969. Grammatica storica della lingua italiana e dei suoi dialetti: Morfologia. Turin: Einaudi.

Department of Italian

University of Bristol

17 Woodland Road

Bristol BS8 1TE

United Kingdom

diego.pescarini@gmail.com 